\title{
IV. Treatment of fibrosis
}

\author{
Chairman: M. 1 . V. JAYSON
}

\section{Failure of corticosteroids to prevent fibrosis}

\author{
E. G. L. BYWATERS \\ From the Royal Postgraduate Medical School, London
}

The big bang of 1949 when Hench and Kendall produced cortisone, the universal and really potent anti-inflammatory agent, still echoes in a few distant canyons but, although everyone agrees on the physiology of this powerful substance, the dramatic effects of big dosage are imbalanced by practical issues like side effects and a long-term inefficacy. If mitral stenosis is due to fibrosis and fibrosis is due to inflammation and prednisone stops inflammation prednisone should prevent the ultimate fibrosis of stenosis, but it does not seem to do so-at least, in the way we use it. Maybe we use it wrongly.

In local scleroderma Allan Dixon and I tried a local approach by local injection and failed to inhibit this local advance, perhaps because we could not keep a local concentration for long enough. In rheumatoid arthritis normal healing of surgical scars by fibrosis is uninhibited with the usual corticosteroid dosage, despite experimental results. While prednisone inhibits fibroblasts in vitro (Nacht and Garson, 1974) and osteoblasts in vivo, collagen production in inflamed joints, replacing and surrounding deposits, seems to progress even on corticosteroids. This failure applies to constrictive pericarditis, to obliterative digital endarteritis, and to the gross fibrosis around old nodules.

Essentially prednisone in large and unphysiological dosage seems to postpone rather than obviate an inflammatory reaction. I suppose if we were able to give it, as in experimental animals, in large amounts over a prolonged period of time it might be effective. Thus hydrocortisone $150 \mathrm{mg} / \mathrm{day}$, given for Dressler's syndrome after a myocardiad infarct, delayed healing, producing by 63 days scar which was estimated at necropsy to be aged 10-14 days with poor healing and aneurysm ${ }^{+}$ formation. This is seen also in many other cases (Bulkley and Roberts, 1974). It is not a practical proposition for treating long-term inflammation although effective in the short run.

Unfortunately most of our problems today are chronic and with us for ever-like inflation, violence, and pollution. I think the same difficulties may be found with proline analogues, but that takes me beyond my brief. 